\title{
Effect of HiLo on skeletal muscle SDH of rats
}

\author{
Qian zhen Yan \\ Department of Physical Education,Wen Shan University, Wen Shan, 663000, China \\ qianzheng@163.com
}

Keywords: HiLo, skeletal muscle, SDH.

\begin{abstract}
Through 4 weeks HiLo training of 72 SD male rats, some very obvious changes of Rat skeletal muscle SDH was found in a quiet state. Some changes of SDH were found immediately after exercise but not obvious, but three hours later after exercise the change of Hypoxia and exercise compound group was more significant. The results show us that rat aerobic metabolism enzyme activity is improved more obviously by Hypoxia and exercise double stimulation.
\end{abstract}

\section{Introduction}

Since the year 1968 Mexico City Olympics, the international sports science once raised the research upsurge of altitude training, but nearly 30 years of theoretical research and practice showed that altitude training in athletes improve exercise capacity, but at the same time, there was also exist many drawbacks[1]. We found that the effect of altitude training is not as high as peoples' expect. The inherent advantages and disadvantages of altitude training, make it become a "double-edged sword" [2]. So people begin to explore an effective method to replace the altitude training, "living high training low"(SDH) is one kind of mode of simulated altitude training. In order to both improve body transport of oxygen and oxygen utilization advantages through efficient use of altitude training hypoxia stimulation, and avoid its negative impact on physical function, SDH theory and method emerged as the times require. It can improve the athletes' capacity and utilization of oxygen transport by hypoxic exposure, and can keep the normal movement of strength training, and the theory and existing studies have demonstrated the effectiveness of HiLo. This research is to study the SDH in the skeletal muscle of rats mainly through the HiLo pattern. It observes the changes of Oxygen metabolism enzyme in rat skeletal muscle of rats, respectively in the quiet state, immediately after exercise and three hours after exercise, to analyse the changes of aerobic sporting ability of rats under the three conditions, and to provide a theoretical basis for scientific training.

\section{The research contents and methods}

The research object. $72 \mathrm{SD}$ male rats, 8 weeks of age, are feed separately according to the experimental animal feeding standard. After one week adaptation training, the rats are randomly divided into 4 groups and take the formal training for 3 weeks. It takes all 4 weeks to train the rats.

Experimental method.1. Experimental groups. After one week adaptation training, the rats are randomly divided into 4 groups: Normoxic control group (C), Normoxic training group (S), HiCo group $(\mathrm{H})$ and HiLo group (HS). And there are 18 rats in each group. 2. Group training arrangement. Normoxic control group (C): normal eating, drinking freely without any training activities. Normoxic training group(S): the rats only take aerobic endurance training every day. HiCo group (H): rats enter the hypoxia house at 8 o'clock at night and slept, and then go out at 8 
o'clock in the next morning. The rats take 12 hours hypoxia training every day, 3 times a week. Hypoxia instrument wil be normal zero before every using, and is set the normal oxygen concentration of $20.9 \%$ to monitor, and oxygen concentration is controled in $15.3 \%$ inside the Hypoxia tent to make a hypoxic environment formed. The simulated altitude is equivalent to $2500 \mathrm{~m}$. HiLo group (HS): rats enter the hypoxia house at 8 o'clock at night and slept, and then go out at 8 o'clock in the next morning. The rats take 12 hours hypoxia training every day, 3 times a week. Hypoxia instrument wil be normal zero before every using, and is set the normal oxygen concentration of $20.9 \%$ to monitor, and oxygen concentration is controled in $15.3 \%$ inside the Hypoxia tent to make a hypoxic environment formed. The simulated altitude is equivalent to $2500 \mathrm{~m}$. And the rats take the aerobic endurance training every day after the hypoxic training at last. Movement pattern: exercise group uses domestic rodents electric treadmill for training to adapt to the peripheral speed of $15 \mathrm{~m} / \mathrm{min}, 30 \mathrm{~min}$ every day, so that the rats can be familiar with treadmill exercise. The load is increased after one week. The speed of $5 \mathrm{~m} / \mathrm{min}$ is increased per week and the time of $10 \mathrm{~min}$ is increased each time. At the last week, the rate of $30 \mathrm{~m} / \mathrm{min}$ and the time of $60 \mathrm{~min}$ will be realized.

Experimental materials. After the end of training program, the rats are anesthetized by $0.4 \%$ sodium pentobarbital, $1 \mathrm{ml} / 100 \mathrm{~g}$ weight. Their musculi gastrocnemius and heart are removed quickly and the heart is weighed in Libra. And then we use the small shear to separate the left and right ventricular, and vernier caliper to measure left ventricular wall thickness, and at last put the musculi gastrocnemius and myocardium into refrigerator of -70 degrees.

\section{Test index}

Succinate dehydrogenase (SDH): in accordance with the Nanjing built succinate dehydrogenase (SDH) test box instructions for testing

Test index steps. First, 200mg myocardial and 200mg right musculi gastrocnemius are weighed accurately and put respectively into the $5 \mathrm{ml}$ small beaker. Second, we take $9 \% 1 \mathrm{ml}$ saline which is cooling advance in the refrigerator(4 degrees), in the beaker with pipettes. Third, we use the Ophthalmology small shear to chop them as soon as possible. (the beaker is in the ice water.) Fourth, the chopped tissue is poured into a homogenate glass tube, and then we use $0.8 \mathrm{ml}$ cold physiological saline to residue the tissue fragments in the beaker and put them together into the homogenate tube to enable the tissue fully homogenized to be $10 \%$ homogenate. Fifth, the homogenate is divided into two parts, one part will make the homogenate prepared by freeze centrifuge centrifugal supernatant, $3000 \mathrm{r} / \mathrm{min} 10 \mathrm{~min}$ to determine SDH activity according to the SDH kit.

Data processing. All the data are based on mean and standard deviation $(X+S D)$ to indicate, using single factor variance analysis method of statistical processing. All data is come out by using the statistical software SPSS in the computer, and significant difference is $\mathrm{P}<0.05$.

\section{Results}

As can be seen from table 1, the changes of SDH in skeletal muscle tissue:

In a quiet state, HiLo group and the Normoxic control group, Normoxic training group, HiCo group had a very significant difference $(\mathrm{P}<0.01)$;

Immediately after the exercise, HiCo group, HiLo group and Normoxic control group had a significant difference $(\mathrm{P}<0.05)$;

Three hours after the exercise, HiCo group, HiLo group and Normoxic control group had a 
significant difference $(\mathrm{P}<0.05)$. There are a significant difference $(\mathrm{P}<0.05)$ between HiLo group and Normoxic training group.

Table 1: Effects of two kinds of models of hypoxia on SDH in skeletal muscle of rats (Unit: U/mgprot)

\begin{tabular}{cccc}
\hline Group & A quiet state & $\begin{array}{c}\text { Immediately after } \\
\text { the exercise }\end{array}$ & $\begin{array}{c}\text { Three hours after } \\
\text { the exercise }\end{array}$ \\
\hline $\begin{array}{c}\text { Normoxic } \\
\text { control group } \\
\text { Normoxic }\end{array}$ & $2.62 \pm 0.23$ & $2.96 \pm 0.88$ & $2.20 \pm 0.89$ \\
training group & $2.85 \pm 0.08$ & $2.42 \pm 1.08$ & $3.18 \pm 0.66$ \\
HiCo group & $3.00 \pm 0.43$ & $4.29 \pm 0.48^{\#}$ & $4.28 \pm 1.16^{\#}$ \\
HiLo group & $4.05 \pm 0.78^{\# \# @ @ \text { 动 }}$ & $4.74 \pm 1.28^{\#}$ & $4.44 \pm 1.25^{\# @}$ \\
\hline
\end{tabular}

\section{Notes:}

Compared to the control group $\mathrm{P}<0.05$, \# showed normal oxygen, \#\# showed $\mathrm{P}<0.01$;

Compared to the training group P $<0.05$, @ showed normal oxygen, @@ showed P $<0.01$;

Compared to the HiCo group $\mathrm{P}<0.05$, is showed normal oxygen, is is showed $\mathrm{P}<0.01$;

Compared to the HiLo group $\mathrm{P}<0.05, \&$ showed normal oxygen, \&\& showed $\mathrm{P}<0.01$;

\section{Analysis and discussion}

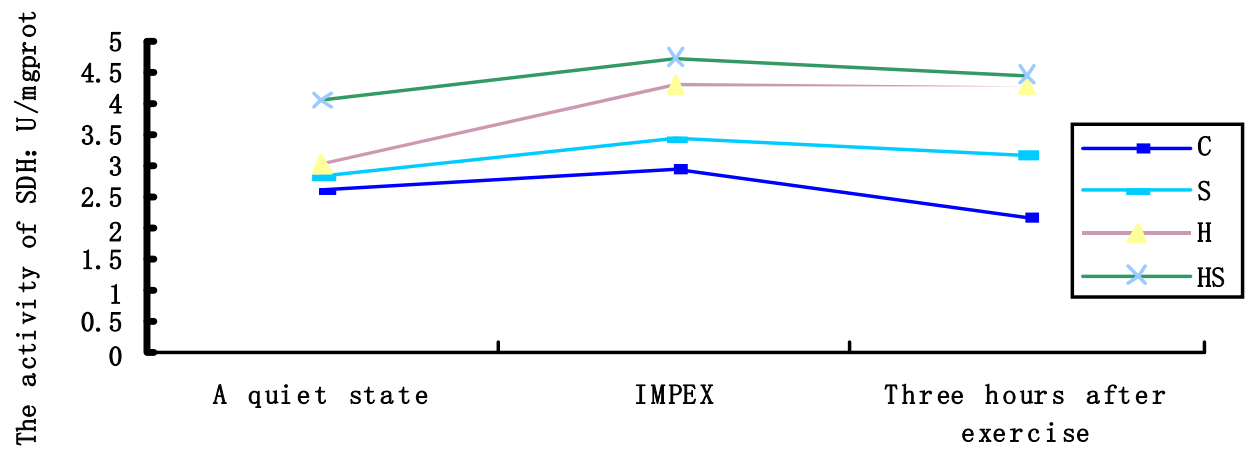

Chart 1 Effect of HiLo on SDH in skeletal muscle of rats

$\mathrm{SDH}$ is the only Mitochondrial intima enzyme added into tricarboxylic acid cycle and is also one of the most important reflect enzyme catalyzed aerobic oxidation ability of the body.It is often used to evaluate the effects of exercise training on aerobic capacity.SDH is in direct contact with the respiratory chain and FADH2 produced by succinic dehydrogenase can be transferred to the iron sulfur center of enzyme, and then enter the respiratory chain.SDH is a key enzyme in tricarboxylic acid cycle, and the level and content of its activity affects tricarboxylic acid cycle speed, and it is the most important way to product energy in the body for a long time during exercise. The muscle gastrocnemius plays an important role in leg movement and consumes more energy, but also need 
more and more energy to supply.Effect of oxygen on the energy is the maximum, so effect of hypoxic factors on the aerobic metabolism to produce energy is relatively large. As can be seen, the implementation of hypoxic training group compared with not the implementation of hypoxic training group, the effect of hypoxic factors on the activity of musculi gastrocnemius SDH is great.

Effect of 4 weeks hypoxia training on SDH activity of rat skeletal muscle is very significant. in a quiet state, there are significant differences $(\mathrm{P}<0.01)$ among Hilo group and the other groups. HiLo group increase 55\% compared to Normoxic control group and increase $42 \%$ compared to the Normoxic control group. These all show that two models of hypoxia for SDH in skeletal muscle of rats increased trend are both obvious after 4 weeks hypoxic training. In the state immediately after exercise, normoxic control group and intermittent hypoxia training group, normoxic training group and intermittent hypoxia training group showed a very significant difference $(\mathrm{P}<0.01)$. There are $45 \%$ increase of the activity of SDH in skeletal muscle in Hico group and $60 \%$ increase in HiLo group respectively. In the state after exercise, normoxic control group and HiCo group, HiLo group; normoxic training group and intermittent hypoxia control group; exercise group and HiCo group showed a significantly difference $(\mathrm{P}<0.05)$. In the recovery period after exercise, whether hypoxia or hypoxia combined exercise, it's obvious to improve the SDH activity of rat skeletal muscle by HiLo. According to the results of the experiment, SDH activity in the musculi gastrocnemius of two hypoxia model is increased under the three conditions, in a quiet, immediately after exercise, and 3 hours after exercise. It shows that 4 weeks Hypoxia training is likely to improve the suitable time of SDH enzyme activity in skeletal muscle of rats.

The experimental results show that, skeletal muscle SDH activity obviously increased after HiLo (though the samples of testing exercise time are few, but it can reflect a trend of increasing to improve the ability of hypoxia training). The author thinks, the reason for elevating the experimental exidase activity relates to the experimental quantitative load. This load can make the muscle cells under appropriate stimulation and cause muscle cell structure and function to produce adaptive changes, make muscle cell oxidase activity increased. Because of enchancing the activity of cell mitochondria oxidase SDH, improving tissue oxygen utilization ability, and conduciving to the skeletal muscle aerobic metabolism, it ensure the energy supplyment of muscle contraction and improve muscle work efficiency and lasting movement ability. Thus after hypoxia training, mitochondrial oxidase activity enhancement is important for improving aerobic capacity. These results show that, hypoxia can cause the increase of SDH activity in rats to a certain extent, and can improve the aerobic capacity and oxidative phosphorylation. The increase of SDH activity induced by Chronic hypoxia may be related to the expression of hypoxia inducible factor 1 (hypoxiainduciblefactor1, HIF-1) upregulation of hypoxia responsive genes (such as a variety of adenylate kinase 3, EPO gene, glycolytic enzymes), thus affecting the metabolism (glycogen synthesis, glucose transport, glucolysis, etc.) HiLo induces SDH activity to be an proceeding upward trend over the time passing. The experiments of Mao Shanshan show that the SDH activity on the seventh day is close to the level of the quiet control group and the SDH activity caused by HiLo is an proceeding upward trend over the time passing. On the twenty-eighth day, SDH activity has reached more than 2 times as the level of the quiet control group, relatively having more lasting effect on improving the aerobic exercise ability. There are reports directly or indirectly support this conclusion, but there are still disputes. Comparison of Levine in altitude training group, HiLo group and normoxic training group of long distance runners $\mathrm{VO}_{2} \mathrm{max}$, average running speed, then we find that normoxic training group of two indicators have no significant change, and the two index levels of group HiLo have some improvement ${ }^{[3]}$. 


\section{Conclusion}

In the Hilo mode, rat skeletal muscle SDH changes significantly in a quiet state, has some changes immediately after exercise, but it is not articularly evident, and in three hours after exercise HiLo group changes more obvious. It shows that Hilo can effectively improve the SDH levels in rat skeletal muscle, in which changes in hypoxia and exercise composite group are more obvious.

\section{References:}

[1] Wilber RL:Sports Med,Vol. 4(2001)No.25, p.249-265.

[2] Q.W.Chen: Liaoning Sport Science and Technology,Vol.6(2005)No.12, p.51-53.’In Chinese"

[3] S.S.Mao,T.B.Pan and R.Y.Wang:Chinese Journal of Sports Medicine,Vol.5(2005)No.24, p.551-554.’'In Chinese" 